Recibido 05 de Enero, 2019 - Aceptado 05 de Febrero, 2019

\title{
Medicina tradicional o complementaria: pacientes que lo usan al mismo tiempo que su tratamiento farmacológico
}

\section{Traditional or complementary medicine: patients who use it at the same time as their pharmacological treatment}

\author{
Richard García ${ }^{1}$
}

\section{RESUMEN}

El objetivo del presente estudio fue determinar el consumo de productos alternativos y complementarios al mismo tiempo que su tratamiento farmacológico en pacientes que adquieren productos en farmacias y boticas de la ciudad de Chiclayo, para lo cual se aplicó un estudio transversal a 400 personas encontrando que el 52\% de los pacientes lo hacen de los cuales solo el $6 \%$ de ellos lo comunican a su médico prescriptor. Además, casi el $100 \%$ de los encuestados manifestaron que vienen consumiendo o lo han hecho en algún momento de su vida, donde el $81 \%$ tienen educación superior.

Palabras claves: Medicina alternativa complementaria, fitoterapia, medicina tradicional

\section{ABSTRACT}

The objective of the present study was to determine the consumption of alternative and complementary products at the same time as their pharmacological treatment in patients who acquire products in pharmacies in Chiclayo city for which a cross-sectional study was applied to 400 people where they found that the $52 \%$ of patients do so, of which only $6 \%$ of them report it to their doctor. In addition, almost $100 \%$ of respondents said they have been consuming or have done so at some time in their lives, where $81 \%$ have higher education.

Keywords: Complementary alternative medicine, herbal medicine, traditional medicine

1. Magíster, Químico Farmacéutico, profesor investigador de Farmacognosia, Fitoterapia y Fitoquímica en la Universidad Alas Peruanas sede Chiclayo, Miembro de la Sociedad Latioamericana de Fitomedicina.

Email: rigarish@gmail.com 


\section{INTRODUCCIÓN}

El uso de la medicina alternativa o complementaria es mundial siendo esta muy importante como movimiento comercial y económico entre los pobladores, se conoce que, en Uganda, por ejemplo, uno por cada doscientas personas usa esta alternativa como tratamiento. El $80 \%$ de la población africana usa la medicina alternativa o complementaria que está incorporado a su sistema de salud(1).

El 80\% de la población de Pakistán recurre a las plantas medicinales para tratarse y el $40 \%$ de los chinos(2) también, mientras que en Sudamérica encontramos que el 79\% de la población de Colombia usan medicina alternativa o complementaria1(1). En China existen 440,700 servicios de salud que brindan atención con medicina alternativa o complementaria (MAC), sumando las camas entre los hospitales de MTC y hospitales generales hacen un total de 520 600. EL 90\% de los hospitales chinos cuentan con un departamento de medicina tradicional(3).

La Organización Mundial de la Salud (OMS) viene promoviendo el uso de la medicina alternativa o completaría a los tratamientos convencionales u alopáticos, alentando las investigaciones científicas que respalden su uso seguro, además, de la difusión del conocimiento(3). Este involucramiento de la OMS permite sumar esfuerzos en la consolidación de un sistema de salud completo e integral, donde se adicionen conocimientos clínicos multidisciplinarios en el paciente que lo necesite.

Según la OMS, la salud "es un estado de completo bienestar físico, mental y social, y no solamente la ausencia de afecciones o enfermedades"(4), comprendiendo no sólo en el tratamiento sintomático específico que muchas veces la medicina convencional y los sistemas de salud se enfocan; sino, la comprensión y el compromiso hacia un tratamiento holístico, completo e integral que se requiere.
En el Perú, la participación de la MAC en el sistema nacional de Salud (EsSalud) es destacable, desde 1998 existe tratamientos alternativos complementarios como la fitoterapia, acupuntura, yoga, entre otros como parte un programa de servicios de medicina alternativa contando con más de 29 centros de atenciones para los asegurados en todo el país.

Gran parte de la medicina tradicional tiene una fuerte conexión con el curanderismo y el misticismo, siendo muchas veces los chamanes quienes logran una curación mediante la administración de brebajes en medio de rituales propios a la cultura ancestral. Muchas teorías se han tejido alrededor de ciertos males inexplicables como la "teoría de la emanación" relacionados a los vientos de alrededores de los cerros; "pérdida del ánimo" referidos al "susto" y "mal del aire"(5).

La gran variedad de plantas que existe en el Perú está basada en la combinación de muchos factores que nos permiten poseer una enriquecida diversidad de climas. Nuestro país presenta 84 eco-regiones de las 107 que existen en el mundo que permite el desarrollo de 4000 plantas nativas y 1400 especies medicinales con 222 domesticadas o semi-domesticada(1).

La deficiente regulación en los productos y servicios en MAC han permitido a varias empresas, que, bajo el concepto de producto natural, vender productos indiscriminadamente como, por ejemplo, mediante nuevos sistemas de mercadeo llamado "redes", donde se gasta más de 13,700 millones de dólares en Norteamérica, de los cuales 4,000 millones de dólares corresponde a multivitamínicos, cifras que promedian la mitad de los gastos anuales en servicios médicos en Estados Unidos (6).

Este descontrolado consumo de productos alternativos sumado a la vasta información publicitaria con alto contenido comercial y la poca cantidad de especialistas en medicina tradicional complementaria vienen creando un problema de salud. Al no existir políticas públicas de com- 
plementariedad entre la MAC y la convencional permite que el paciente elija su "mejor tratamiento" basada en una información sesgada (7).

Según María Duarte del Instituto de Salud Pública de México, la complementariedad entre la MAC y la convencional si es posible con una política inclusiva de salud pública por parte del estado, donde regule los productos naturales con leyes que controlen el comercio y la publicidad informal de estos productos, como la implementación de sistemas de salud mixtos con especialistas complementarios e integrales (7).

En el Perú, a través de uno de los brazos del Ministerio de Salud, INMETRA (Instituto Nacional de Medicina Tradicional) viene realizando esfuerzos por vincular la medicina tradicional o complementaria con la medicina convencional o académica, promoviendo el uso responsable y profesional en el uso de plantas medicinales en la atención primaria de salud (8).

Existe una falta de confianza en la comunicación por parte de los pacientes que usan MAC y que al mismo tiempo visita un centro de salud convencional, un estudio en la Clínica Pediátrica del Hospital Italiano de Buenos Aires, concluyó que el $84.5 \%$ de los pacientes encuestados conocía algún tipo de medicina alternativa o complementaria, seguían el tratamiento a la par del alopático, pero el $42 \%$ no informó al médico de cabecera (9).

Esta falta de confianza de los pacientes que usan medicina alternativa para comunicar a su médica tratante en una consulta se debe al bajo nivel de conocimiento que tienen los profesionales con estos temas. En un estudio realizado en Colombia vinculados al departamento de Cundinamarca arrojó que el 93\% de los médicos encuestados tiene desconocimientos en MAC, sin embargo el $41 \%$ desea capacitarse en estos temas(10).

\section{MÉTODOS Y MATERIAL ES}

El estudio es de corte transversal y descriptivo, donde se incluyen a clientes que compran en las principales boticas y farmacias de la ciudad de Chiclayo. La muestra es de 400 personas, compradores, hombres o mujeres, tomadas en forma consecutiva y por conveniencia, mayores de edad.

El instrumento usado es una encuesta, no validada y consta de 10 preguntas. La toma se dio de forma anónima, para obtener una información más sincera, con la finalidad de conocer si usa medicina alternativa complementaria, tipo, frecuencia, adquisición, información al médico, satisfacción, reacciones no deseadas, además de su procedencia, nivel de educación y vivienda.

\section{RESULTADOS}

Los resultados muestran que el 52\% de los clientes están consumiendo medicina tradicional o complementaria junto al tratamiento médico convencional. De los cuales el 31\% corresponde a las mujeres y el $21 \%$ a los hombres.

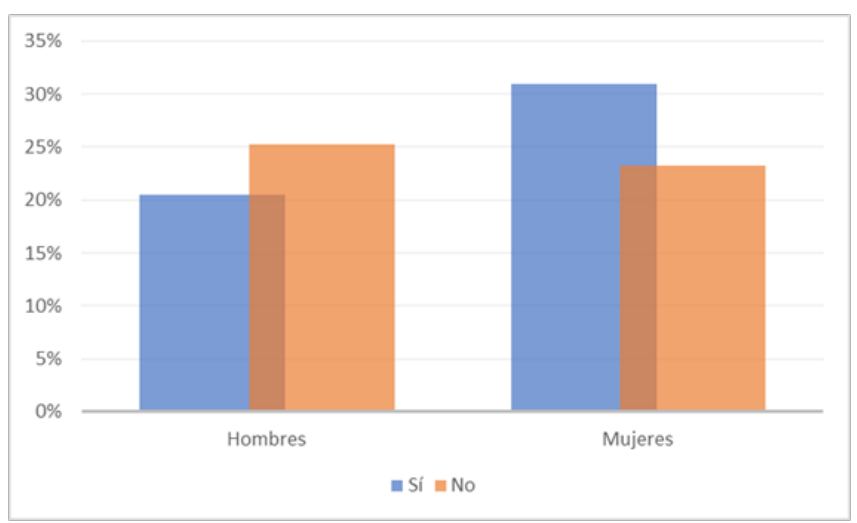

Figura No 1 . Personas (\%) que está usando medicina complementaria al mismo tiempo que su tratamiento médico convencional 
Sólo el 6\% de las personas que consumen MAC comunica a su médico de lo que viene haciendo.

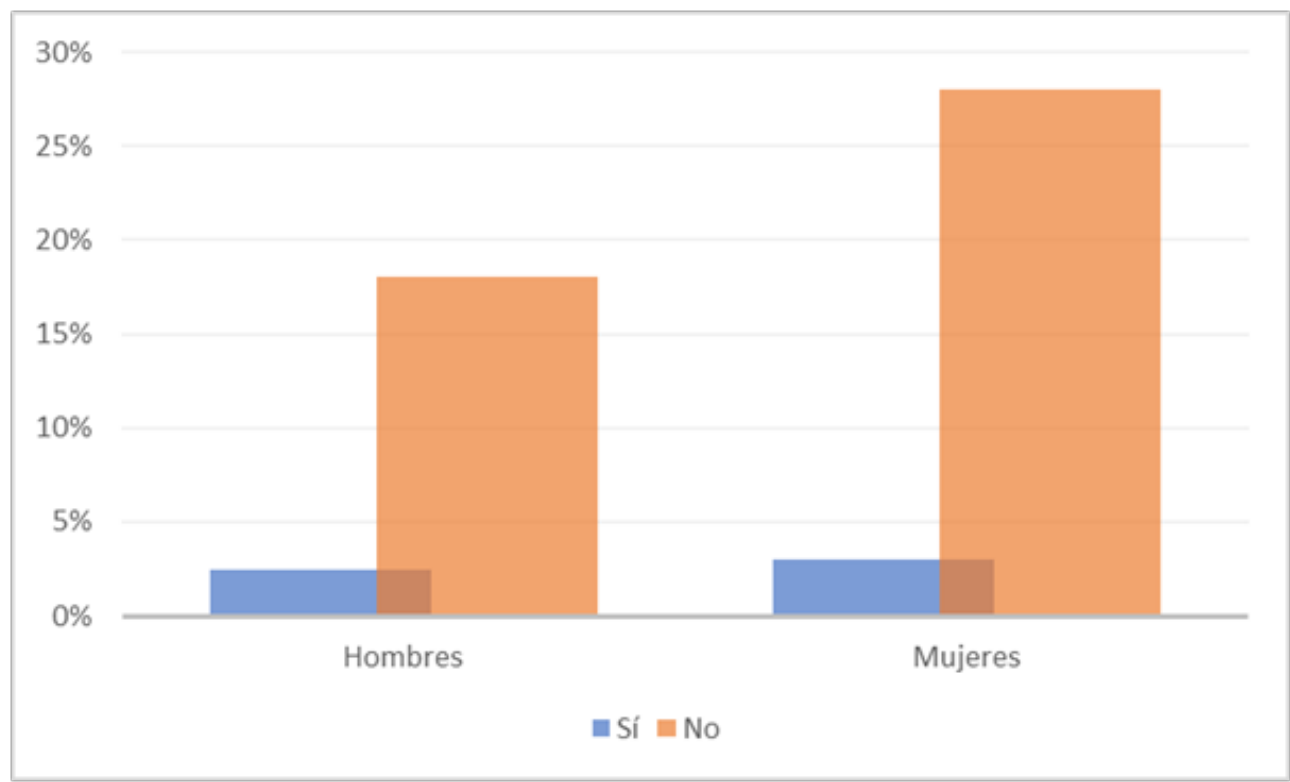

Figura No 2. Personas (\%) que consumen medicina tradicional o alternativa y que comunica a su médico.

Casi el 100\% de los encuestados manifestaron que consumen o han consumido algún tipo de MAC, siendo los más comunes las fitomedicamentos.

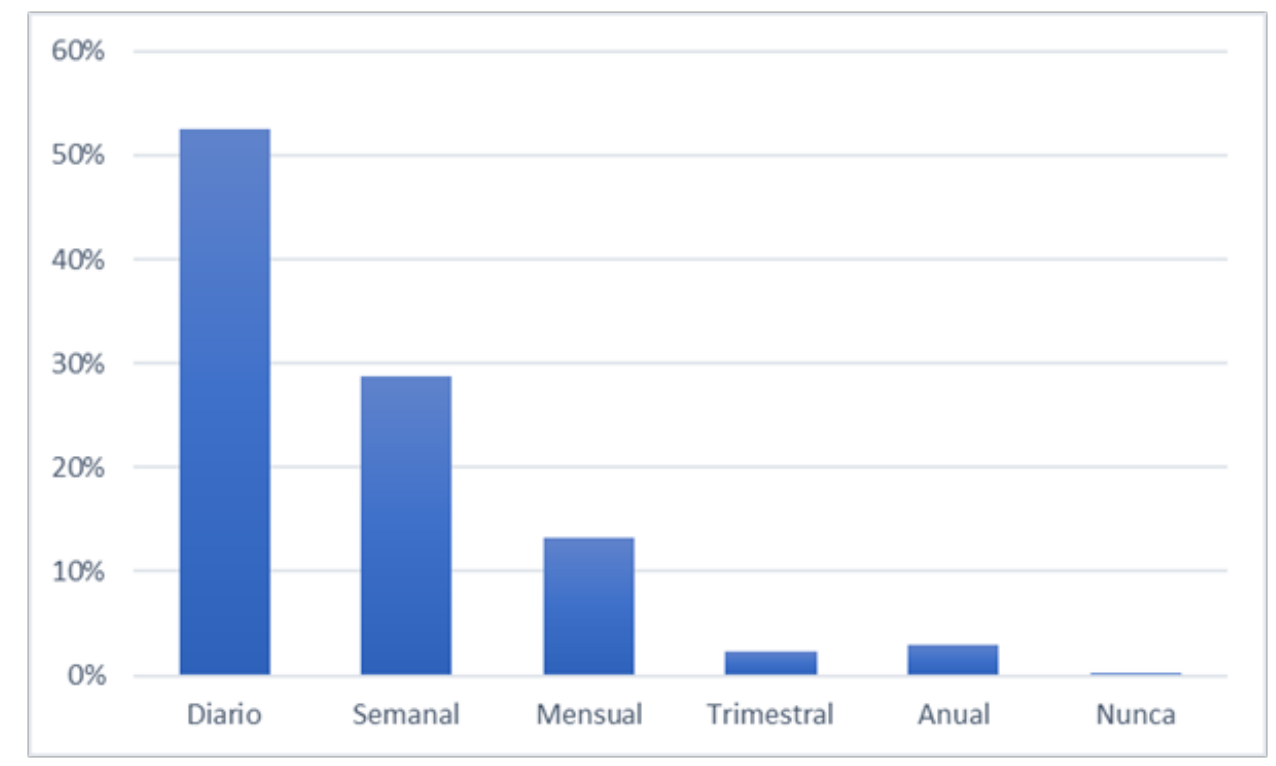

Figura $N^{0} 3$. Frecuencia de consumo de medicina natural o alternativa 
El 81\% de los encuestados que afirmaron consumir o haber consumido MAC tiene una profesión superior, siendo $45 \%$ para profesionales de instituto y $36 \%$ para los egresados de una universidad.

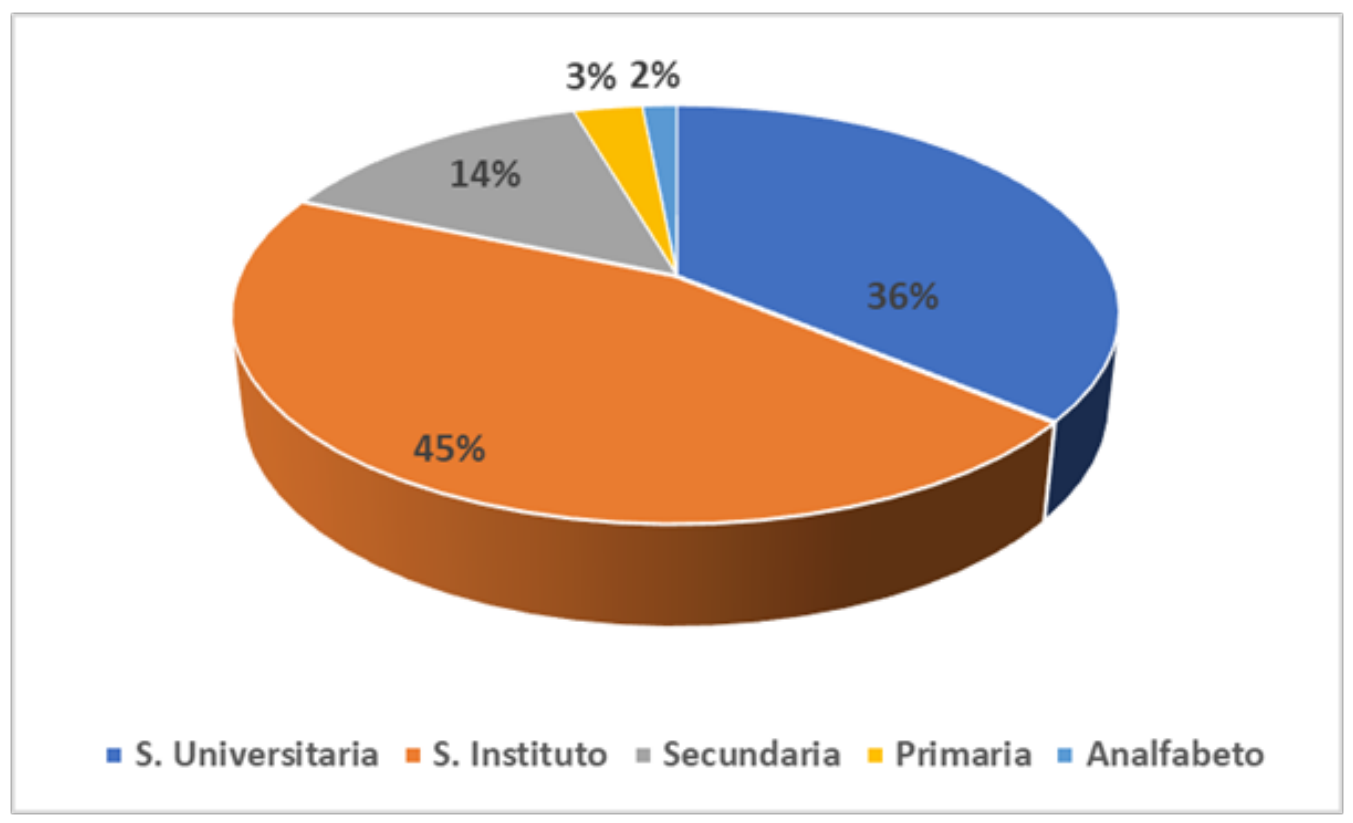

Figura $\mathrm{N}^{\circ} 4$. Niveles educativos de los encuestados que consumen o han consumido medicina alternativa o complementaria

\section{DISCUSIÓN}

Chiclayo es una ciudad con muchas posibilidades de obtener medicina alternativa, quienes lo prefieren pueden optar por ir al mercado modelo, que tiene más de 80 puestos con productos naturales, según un estudio de la antropóloga italiana, Adine Gavazzi, afirmó que se venden más de 1000 variedades de plantas; muchos otros los consiguen de las diferentes casas naturistas que están ubicadas en muchas de las zonas del centro, las boticas y farmacias también venden productos fitoterapeúticos.

Dentro de la medicina alternativa, también tenemos a la homeopatía, acupuntura, digitopuntura, reflexología, quiropraxia, medicina ayurveda, reiki, ventosas, entre otros, que también son frecuentado por los chiclayanos, con la dificultad de encontrar especialistas en dichas ramas.

Según los resultados, el 52\% de las personas que reciben un tratamiento convencional también consume MAC (Figura 1), esto se presenta como un gran desafío para los involucrados en la atención de salud, ya que el profesional prescriptor debe de tener los conocimientos necesarios para manejar casos de interacciones entre productos fitoterapeuticos y académicos, por ejemplo, las interacciones entre el ajo y la aspirina, el té verde y el hierro, la manzanilla y los antagonistas del calcio, etc.

Muchos de los fármacos químicos tienen la misma vía de metabolización que las plantas medicinales, de esta manera pueden bloquear, disminuir o incrementar la actividad de bioquímica produciendo reacciones indeseables o efecto reducido. El sistema de citocromo P-450, es uno de los principales encargados de activar o desactivar muchos fármacos o sustancias extrañas con el objetivo de oxidar y convertirla en estructuras más hidrosolubles para su posterior eliminación

Sin embargo, a pesar del alto consumo de productos alternativos o complementarios al mismo tiempo que el tratamiento convencional, sólo el $6 \%$ comunica de esto a su médico prescriptor 
(Figura 2), adquiriendo el tratamiento un alto riesgo de interacción. Está poca confianza para comunicar lo que viene consumiendo el paciente puede deberse a muchos factores, como, por ejemplo, el alto grado de desconocimiento en temas de productos alternativos o complementarios como el estudio visto por Eymanna, donde el 93\% de los médicos desconocían estos tratamientos(10) o poca empatía y capacidad para establecer una buena relación entre tantos.

Casi el $100 \%$ de los encuestados manifestaron haber consumido medicina alternativa o complementaria (Figura 3) en algún periodo de su vida, de los cuales el $81 \%$ son profesionales con estudios superiores (Figura 4), quienes lo consumieron lo hicieron con una frecuencia muy elevada, esto representa un gran responsabilidad para los gobiernos de instaurar políticas serias de integración entre ambas corrientes y control en la venta indiscriminada de productos naturales, atenciones de medicina alternativas o complementarias sin ningún control, hasta inclusive la venta de productos farmacéuticos de uso restrictivo sin la debida receta médica.

\section{CONCLUSIONES}

Más del 50\% de los encuestados en la ciudad de Chiclayo, afirmaron que consume medicina alternativa o complementaria al mismo tiempo que el fármaco de prescripción médica.

Sólo el 6\% de los encuestados manifestaron que sí comunican a su médico prescriptor el consumo de medicina alternativa o complementaria al mismo tiempo que su tratamiento farmacológico.

Casi el 100\% de los encuestados manifestaron su consumo de medicina natural o alternativa en algún momento de su vida, de los cuales el $81 \%$ tienen estudios superiores.

\section{REFERENCIAS BIBLIOGRÁFICAS}

1.Bussmann R., Sharon D., Plantas medicinales de los Andes y la Amazonía. Primera edición, Graficart SRL. Perú. 2016 p7

2.Santillo H., Hierbas. La Curación Natural. Edit. Subhuti Dhramanada. Grupo editorial Tomo, S.A. de C.V. 2001. 585 pp.

3.Organización mundial de la salud. [Internet]. Estrategia de la OMS sobre la medicina tradicional 2014-2023. $22 \mathrm{Fe}-$ brero 2019. Recuperado de https://apps.who.int/iris/bitstream/handle/10665/95008/9789243506098_spa.pdf;jsessi onid=2F6DBE92ED018C6BA5F06FA26670BEA6? sequen $\mathrm{ce}=1$

4.Organización mundial de la salud. [Internet]. Estrategia de la OMS sobre la medicina tradicional 2014-2023. $22 \mathrm{Fe}$ brero 2019. Recuperado de https://www.who.int/suggestions/faq/es/

5.Alonso J, Curso anual de fitomedicina. Metabolismo I Diabetes. Buenos Aires. Argentina. 2018;1.8; 39.4p.

6.Eisenberg DM, Kessler RC, Foster C, Norlock FE, Calkins DR, Delbanco TL. Unconventional medicine in the United States - prevalence, costs, and patterns of use. N Engl J Med. 1993;328:246-52

7.Duarte M., Medicina occidental y otras alternativas: ¿es posible su complementariedad? Reflexiones conceptuales. Cad. Saúde Pública, Rio de Janeiro, 19(2):635-643, marabr, 2003

8.Cañigueral S, Dellacassa E, Bandoni A, Plantas Medicinales y Fitoterapia

9. Indicadores de Dependencia o Factores de Desarrollo?. Acta Farm. Bonaerense 2003;22 (3): 265-78

10.Eymanna A, Bellomoa M, Catsicarisav C, Wahrena C, Utilización de medicina alternativa o complementaria en una población pediátrica de un hospital de comunidad. Arch Argent Pediatr 2009;107(4):321-328

11.Sierra-Ríos J, Urrego-Mendoza D, Jaime-Jaimes J, Conocimientos, actitudes y prácticas acerca de la medicina alternativa en médicos vinculados a hospitales públicos de Cundinamarca, Colombia. Rev. salud pública. 2012;14 (3): 478-490 